\title{
PLEBISCITO DE LA PAZ: \\ PARTICIPACIÓN ELECTORAL DE LOS MIGRANTES COLOMBIANOS EN GUAYAQUIL (ECUADOR)
}

\author{
Plebiscite of Peace: voter turnout \\ of the Colombian migrants in Guayaquil (Ecuador)
}

\author{
Sebastián Umpierrez de Reguero* \\ Paula Nimbriotis Manzur ${ }^{* *}$ \\ Germán Campos-Herrera** \\ Ingrid Ríos Rivera***
}

\begin{abstract}
Resumen. Este artículo ayuda a contextualizar el Plebiscito de Paz de 2016. Mejora el entendimiento sobre el voto en el exterior en mecanismos de democracia directa. Con datos observacionales, se realizan varios ejercicios estadísticos. A nivel descriptivo, se agrupan los motivos para (no) votar en el Plebiscito de la Paz en percepciones positivas y negativas. En el análisis inferencial, se reporta que las percepciones positivas se asocian significativamente a la participación electoral en el exterior. Esta participación se correlaciona positivamente a la experiencia en elecciones anteriores en Colombia, al seguimiento del Acuerdo de Paz y a la preocupación por las elecciones de 2018. Adicionalmente, la evidencia empírica subraya que los jóvenes son quienes tienen mayores probabilidades de participar en elecciones desde el exterior, de alta relevancia histórica como las elecciones de octubre de 2016.
\end{abstract}

Palabras clave: voto extraterritorial; Plebiscito de la Paz; migrantes; refugiados; Colombia; Ecuador.

Abstract. This article aims to contextualize the Peace Plebiscite of 2016. It improves the understanding of external voting on mechanisms of direct democracy. With observational data, several statistical exercises are carried out. On a descriptive level, the reasons for (not) voting in the Plebiscite of Peace are grouped into positive and negative perceptions. In the inferential analysis, we report that positive perceptions are significantly associated with emigrant voter turnout. This type of turnout positively correlates with the experience in previous elections (in Colombia), the follow-up to the Peace Agreement and the concern for the 2018 elections. In addition,

\footnotetext{
* Universidad Diego Portales. Santiago, Chile / Universidad Casa Grande. Guayaquil, Ecuador.

** Universidad Casa Grande. Guayaquil, Ecuador.

${ }^{* * *}$ Universidad Diego Portales. Santiago, Chile.

${ }^{* * * *}$ Universidad de Chile. Santiago, Chile / Universidad Casa Grande. Guayaquil, Ecuador.
} 
empirical evidence highlights that young people are the ones most likely to participate in elections from abroad, with high historical relevance as the elections of October 2016.

Keywords: extraterritorial vote; Plebiscite of Peace; migrants; refugees; Colombia; Ecuador.

\section{Introducción}

La literatura sobre la extensión de derechos políticos para los ciudadanos no-residentes ha tenido un crecimiento lineal (IDEA-IFE, 2007; Lafleur, 2013). América Latina no se ha quedado atrás en relación a este reconocimiento. Actualmente, la práctica del voto extraterritorial es habitual en la región, salvo contados casos como Cuba, Nicaragua o Uruguay (Palop-García, Pedroza, 2016). En 1961, Colombia fue el primer país latinoamericano en legislar este derecho (Bermúdez, Lafleur, 2015).

A pesar de haber académicos que aseveran que el tema del voto extraterritorial ha sido poco analizado (Lafleur, 2015), los estudios existentes, tanto normativos como empíricos, son extensos y variopintos. Existe un número creciente de aportes que lo vinculan con: nuevas nociones de ciudadanía (Bauböck, 2007; Collyer, 2014a); instituciones del país de origen (Gamlen, 2015; Lafleur, 2013, Østergaard-Nielsen, Ciornei, 2017; PalopGarcía, 2017); patrones de adopción (Erlingsson, Tuman, 2017; Lisi et alii, 2014; Rhodes, Harutyunyan, 2010; Turcu, Urbatsch, 2014); democracia/ autoritarismo (Brand, 2010; Collyer, 2014b); hasta con percepciones políticas (Boccagni, 2011; Escobar, Arana, McCann, 2014; Umpierrez de Reguero, Dandoy, Palma, 2017). Sin embargo, no se ha precisado de manera específica en las diferencias que podrían emerger por tipos de elecciones. En efecto, los trabajos previos se centran en elecciones presidenciales y legislativas, dejando de lado los referéndums desde el exterior.

De ahí que en este artículo se busque explorar la relación entre el voto extraterritorial y el referéndum. En particular, se pretende hallar los determinantes de votación en un referéndum e identificar los motivos más frecuentes para (no) votar en este proceso electoral desde el exterior. En este sentido, la revisión de la literatura examina las principales contribuciones del tema sobre el voto extraterritorial y se postulan hipótesis a partir de la conceptualización de las prácticas políticas transnacionales en migración.

El diseño de investigación empleado es observacional, de tipo descriptivo y explicativo. Los datos recolectados sobre el Plebiscito de la Paz del 2 de octubre de 2016, provienen de 590 respuestas de migrantes y refugiados colombianos en Guayaquil (Ecuador). En efecto, las variables con las que se construye la evidencia empírica, se articulan a partir de las siguientes tres dimensiones: (1) perfil socio-demográfico, (2) compromiso 
migrante y (3) comportamiento electoral. Sobre esta base, se contribuye al acervo bibliográfico que ayuda a contextualizar el Plebiscito de Paz del 2 de octubre de 2016 y mejora el entendimiento sobre el voto extraterritorial en mecanismos de democracia directa.

Los motivos de elegir el Plebiscito de la Paz del 2 de octubre de 2016, abordado desde la perspectiva de los colombianos residentes en Guayaquil (Ecuador), se estructuran a partir de justificaciones académicas, de relevancia histórica y contingencia política. Lo habitual es examinar los procesos de inmigración desde la óptica de las sociedades de acogida más emblemáticas o demográficamente más concurridas (i.e. Estados Unidos). Ecuador, en este sentido, no cumple con estas características -las migraciones históricas de colombianos tienen como principales destinos Estados Unidos, Venezuela y España (en orden de importancia demográfica) (Naciones Unidas, 2015). Sin embargo, si se considera a la categoría de refugiados, se debe repensar la selección de caso Ecuador, hoy en día, es uno de los mayores receptores de refugiados colombianos en comparación con otros países de América Latina (ACNUR, 2017).

Las relaciones bilaterales entre Colombia y Ecuador han tenido ocasionales impases como el conflicto de Angostura. No obstante, en el ámbito de la migración, tanto ecuatorianos como colombianos, han cruzado las fronteras repetidas veces para quedarse en el país vecino sin mayores ataduras. Principalmente, dicha situación se ha encontrado asociada a oportunidades económicas (sobre todo desde la adopción del dólar estadounidense en Ecuador) pero también por circunstancias vinculadas, directa e indirectamente, al conflicto armado colombiano (Carreño-Malaver, 2014). De hecho, el Plebiscito de la Paz del 2 de octubre de 2016 fue un evento histórico para Colombia y América Latina.

"¿Apoya el acuerdo final para la terminación del conflicto y construcción de una paz estable y duradera?" (Alto Comisionado Para La Paz, 24 de noviembre de 2016). Con esta pregunta, el presidente Juan Manuel Santos convocó a las urnas a los ciudadanos colombianos, dentro y fuera del país, a refrendar el acuerdo de paz entre su gobierno y las Fuerzas Armadas Revolucionarias de Colombia (FARC). Este intento no resultó como el gobierno esperaba. Él "así no" primó sobre los que apostaron al esfuerzo gubernamental.

Tabla 1. Datos agregados de la participación (total) electoral en el Plebiscito de la Paz

\begin{tabular}{c|c|c}
\hline & Votos válidos & $\%$ \\
\hline Sí & 63.773 .482 & 49,8 \\
\hline No & 6.431 .376 & 50,2 \\
\hline
\end{tabular}

Votación total: 13.006 .047 de 34.899 .945 personas registradas $(37,4 \%)$. Elaboración propia. Fuente: Registraduría Nacional del Estado Civil (2017). 
Este artículo se estructura en cinco apartados adicionales a esta introducción. Se comienza referenciando brevemente los aportes y limitaciones de los estudios sobre el voto en el exterior. Luego, se formulan las hipótesis que dan paso a la discusión metodológica. Posteriormente, se detallan las fuentes, tipos de medición e identificación de las variables de interés, así como se selecciona los métodos de análisis para llevar a cabo la sección empírica. En consecuencia, en el siguiente apartado se presentan y se discuten los resultados.

\section{Marco teórico e hipótesis}

El voto extraterritorial, externo o en el exterior, forma parte de un amplio conjunto de políticas diaspóricas (Lafleur, 2011) que son especialmente difíciles de separar de las prácticas políticas transnacionales (Collyer, Vathi, 2007; Østergaard-Nielsen, 2003). La literatura existente, a pesar de ser todavía reducida, tiene importantes contribuciones tanto normativas (Barry, 2006; Bauböck, 2007; López-Guerra, 2005; Rubio-Marin, 2006) como empíricas (Belchior et alii, 2017; Boccagni, 2011; Collyer, Vathi, 2007; Escobar, Arana, McCann, 2014; Gamlen, 2015; Rhodes, Harutyunyan, 2010; Turcu, Urbatsch, 2014). No obstante, todavía es limitado el estudio de los efectos del voto extraterritorial en referéndums. Uno, sino el único, de los textos al respecto, analiza el comportamiento electoral transnacional en las elecciones ecuatorianas de referéndum y consulta popular de 2011 (Boccagni, Ramírez, 2013).

La adopción del voto extraterritorial es progresiva (Lafleur, 2015). Los países extienden derechos de participación política a sus emigrantes, ya sea en elecciones presidenciales, legislativas, supra-nacionales e inclusive de referéndum y sub-nacionales. De hecho, un aproximado de 31 Estados aceptan la incorporación de sus emigrantes para el voto en referéndum (IDEA-IFE, 2007). En América Latina, solo cuatro países otorgan a sus ciudadanos no-residentes derechos para participar en este tipo de elecciones: Chile, Colombia, Ecuador y Perú. Colombia es pionero al respecto -al permitir que sus ciudadanos residentes en el exterior puedan votar en plebiscitos de carácter nacional a partir de la Constitución de 1991 en adelante-.

La extensión de derechos de participación política transnacional no guarda necesariamente relación con la idea de democratización de los países de origen (Lafleur, 2015; Rhodes, Harutyunyan, 2010). En el caso de países latinoamericanos como Argentina y Colombia, la adopción del voto extraterritorial en sus marcos jurídicos efectivamente sucede en un contexto de transición política (Calderón-Chelius, 2006); sin embargo, Argelia o Mozambique son evidencias para dudar de una relación significativa, no espuria, con la democratización (Collyer, 2014b).

El voto extraterritorial no solo tiene como propósito el incrementar la participación política de los emigrantes y por ende, construir confianza en 
los procesos electorales de los Estados de origen (IDEA-IFE, 2007); también forma parte de los debates académicos sobre su relación con la soberanía (Collyer, 2014b), las afectaciones producidas en las instituciones políticas del país de origen (Gamlen, 2015; Lafleur, 2013) o inclusive, para discutir sobre la vigencia del concepto de ciudadanía westfaliana, atada al Estado-Nación (Baübock, 2007; Collyer, 2014a).

\subsection{Perfil socio-demográfico}

La noción de "estatus socio-económico" hace referencia a la educación y a los ingresos del individuo como factores que influyen en la participación, en la política y en las preferencias electorales, a distintos niveles y contextos. El perfil socio-demográfico indica que existen rasgos comunes en relación a la edad (e.g. 25-50 años), y la procedencia principalmente urbana (Aysa-Lastra, 2006; Bermúdez, 2014). En el estudio enfocado en el caso colombiano propuesto por Escobar, Arana y McCann (2014), la educación resultó ser una variable determinante en el perfil socioeconómico del expatriado, donde el nivel educativo es más elevado en comparación con la población general en Colombia.

Por su parte, "las actividades transnacionales, (...) no son propias de los inmigrantes más pobres y marginalizados, sino que están asociadas de manera consistente con mayores recursos de capital humano: más escolaridad, más años de experiencia (en el país de acogida), mayor estatus ocupacional" (Portes, 2005, p. 12). Sin embargo, tomando en consideración el contexto de salida y recepción, la participación del migrante se ve influenciada por una necesidad de adaptación, esencialmente económica (ibidem).

La procedencia de áreas urbanas parece ser un indicador común asociado al perfil del migrante colombiano. Incluso, su futuro lugar de asentamiento en el país de acogida, se puede vincular a la región a la que el migrante pertenecía en el país de origen y a su clase social (Garay-Salamanca, Medina, 2007). En cuanto a la participación política en el ámbito electoral, se menciona como ejemplo el caso de los inmigrantes de zonas urbanas en un contexto de violencia que buscan adaptarse e integrarse más rápido en los lugares de acogida y tienden a evitar la participación activa en la sociedad de origen (Guarnizo, Sánchez, Roach, 1999).

La migración y el transnacionalismo, al ser procesos influenciados por el género (Boccagni, 2011; Boccagni, Ramírez, 2013; Giorguli-Saucedo, Itzigsohn, 2006) determinan características significativas del perfil sociodemográfico del migrante. Incluso, existen roles específicos según el género que se relacionan a los empleos y a la participación transfronteriza. Los hombres se encuentran, mayoritariamente, en cargos empresariales y de activismo político (Portes, 2005). Por otra parte, la participación transnacional en el movimiento 
y participación electoral, se ve protagonizada por mujeres, puesto que hay una mayoría femenina en las cifras de la población migrante colombiana; pero no existe diferencia en cuanto a su estado civil (Aysa-Lastra, 2006).

La participación de colombianos no-residentes se ve influenciada por características del perfil socio-demográfico que guardan relación con un tiempo sustancial de residencia en el país de acogida. La cantidad de años vivida en el exterior tiene incidencia en las relaciones transnacionales existentes entre los migrantes (Boccagni, Ramírez, 2013). Un ejemplo claro de la participación en el exterior son las actividades sociales y de filantropía, especialmente cuando existe una situación de emergencia (Portes, Escobar, Walton, 2006; Bermúdez, 2014; Clavijo-Padilla, 2013; Umpierrez de Reguero, GonzálezParedes, Jara-Alba, 2018). La creciente atención sobre los colombianos en el exterior, responde a un contexto de esfuerzos gubernamentales por incentivar una conexión con el país de origen y promover su participación económica, social y política (Bermúdez, Lafleur, 2015). Presentados estos argumentos, esta subsección asume la siguiente hipótesis:

H1: Los colombianos residentes en Guayaquil de (a) procedencia urbana, (b) género femenino, (c) que tienen menos de 10 años de residencia, y (d) se encuentran entre los 25 a los 50 años de edad, tienen mayores probabilidades de votar en el Plebiscito de la Paz.

\subsection{Compromiso migrante}

En el caso colombiano, la doble ciudadanía sustenta la pertenencia al país de origen y los vínculos transnacionales, que no se oponen a la participación en el país de destino (Escobar, 2006). Sin embargo, existen algunos efectos a considerar sobre la doble ciudadanía. En primer lugar, la idea de democracia ejercida por ciudadanos con doble nacionalidad podría ser calificada como un vínculo vacío, nacional y cívico (Renshon, 2001). En respuesta, es pertinente distinguir entre doble nacionalidad y doble ciudadanía, pues implican distintos niveles de compromiso: el primer concepto hace referencia a los vínculos que se mantienen con la comunidad, lo que implica una forma de ver el mundo, sus prácticas y las conexiones emocionales existentes; mientras que la doble ciudadanía, engloba derechos políticos, económicos y sociales (ibidem).

Se trata entonces de derechos y obligaciones que -como ciudadano- se deben cumplir al ser parte de la esfera política; además de que las decisiones tomadas no tendrían efectos directos sobre el diario vivir del ciudadano no-residente (Bauböck, 2003). No obstante, desde la perspectiva transnacional, la adaptación política al país de acogida, no implica una exclusión en el involucramiento político en el lugar de origen (Levitt, De La Dehesa, 2003; Levitt, Glick-Schiller, 2004). Más bien, la doble ciudadanía se convierte en el instrumento legal para permitir que se mantengan los vínculos con el origen del 
migrante (Escobar, 2007). Incluso, las prácticas transnacionales de migrantes y refugiados pueden variar dependiendo de los recursos y políticas disponibles en el país de residencia (Østergaard-Nielsen, 2003).

De ahí que exista un componente simbólico importante en relación a la visión de la ciudadanía del emigrante, la misma que se encuentra concebida como herramienta de construcción de sentimientos hacia la nación e identidad (Barry, 2006). Se discute sobre el (des)interés por mantenerse vinculados con la política del lugar de origen, que también se puede entender como un gradiente de desconfianza provocada por los procesos políticos existentes (González, 2010), haciendo énfasis en las limitaciones a las que los migrantes se enfrentan al momento de ejercer su participación. Por otra parte, existe también un índice prolongado de baja participación política que probablemente está protagonizado por el desinterés del sistema político colombiano a nivel nacional (Guarnizo, Portes, Haller, 2003).

La inclusión de los migrantes en las elecciones fue una respuesta del gobierno colombiano ante la salida al exterior de un número significativo de sus ciudadanos. Esto contribuyó como una medida para darle legitimidad al sistema electoral en Colombia (González, 2010). A pesar de que los emigrantes no habían sido un grupo influyente en la política nacional, su rol fue incrementando e interpretándose como un mecanismo para mantener la lealtad con Colombia (Roberts, Frank, Lozano, 1999). Sin embargo, aunque las medidas gubernamentales parezcan la razón primordial del transnacionalismo; éste último empieza como iniciativa de los propios emigrantes que buscan crear redes que los conecten con su país de origen (Portes, 2005). Un aspecto que puede llegar a influenciar en el desarrollo de actividades transnacionales en comunidades emigrantes es "una recepción hostil de las autoridades y la ciudadanía de la sociedad receptora" (ibidem).

El interés por la agenda enfocada en derechos humanos y resolución de conflictos, entre tanto, también ha sido una motivación para dar paso a la participación migrante. Es creciente la incorporación de actores no-estatales en problemáticas locales, nacionales e internacionales. La participación ya no es exclusiva de los gobiernos sino que la esfera de acción se ha ampliado para organizaciones civiles -los grupos/redes transnacionales han logrado ejercer presión en la agenda política- (Ardila, 2009). La participación política de los emigrantes va más allá de su involucramiento en los procesos electorales (Østergaard-Nielsen, 2016; Palop-García, 2017; Ragazzi, 2014).

Estudios demuestran que existe una conexión entre el compromiso migrante y la pertenencia a organizaciones civiles (Boccagni, Ramírez, 2013; Guarnizo, Portes, Haller, 2003; Martiniello, Lafleur, 2008). De las organizaciones civiles a las cuales pertenecen los emigrantes, destacan aquellas que tienen mayor cantidad de afiliados (Boccagni, Ramírez, 2013). En el caso 
colombiano, se debe resaltar las que se relacionan con el Acuerdo de Paz entre el gobierno y las guerrillas (Bermúdez, Lafleur, 2015). Por ejemplo, el Foro Internacional de Víctimas busca el involucramiento tanto de hombres y mujeres en el proceso de paz. No obstante, la participación de estos actores no-estatales ha dependido de los representantes políticos de turno, pues no existen medios eficaces y perennes de consulta a la sociedad (Ardila, 2009). En relación a esta sub-sección, se asume la siguiente hipótesis:

H2: Los colombianos residentes en Guayaquil (a) que no han obtenido la ciudadanía ecuatoriana, (b) que se encuentran afiliados a un partido político colombiano, (c) que pertenecen a una asociación civil de migrantes, y (d) que desean retornar a Colombia, tienen mayores probabilidades de votar en el Plebiscito de la Paz.

\subsection{Participación electoral transnacional}

La participación electoral en relación al compromiso migrante está vinculada a sentimientos de apego al país de origen (Boccagni, 2011). Estas actitudes son el resultado de una mezcla de patriotismo y a la vez, rechazo a la política nacional (ibidem). De manera que al abordar el transnacionalismo con sus instituciones y nexos políticos, económicos y socio-culturales de origen, existe una influencia considerable para quienes migraron, quienes aun piensan retornar y quienes se quedaron atrás (Levitt, Waters, 2002). El concepto de transnacionalismo, que originalmente implicaba esfuerzos y acciones para ser parte de "vidas cotidianas en el extranjero" (Portes, Escobar, Walton, 2006), ahora involucra participación política, económica y social.

La participación de transmigrantes no se había considerado como prioritaria hasta la década de 1990. La percepción de los gobiernos latinoamericanos giraba en torno a una imagen asociada a traición cívica, malas situaciones económicas y falta de oportunidades laborales (Berg, Tamagno, 2006). Los ciudadanos no-residentes en las últimas dos décadas han logrado ser considerados como parte de los grupos de influencia de la sociedad civil (Ardila, 2009). Bajo este contexto, los comportamientos de los migrantes colombianos en referencia a la participación política, suelen replicarse pues quienes participaron de manera activa en su país de origen, generalmente repiten este patrón en el país de residencia (Guarnizo, Portes, Haller, 2003). Además, aquellos migrantes, cuyas familias esperan su retorno, tienden a participar de manera más activa en actividades sociales, económicas y políticas (Portes, 2005).

Existen varios factores que tienen influencia en el interés del Estado colombiano por el voto en el exterior como: el creciente envío de remesas económicas, la relación entre el tema migratorio y la seguridad nacional, la preocupación por la fuga de cerebros, el deseo de mejorar la imagen 
internacional del país y el rol de los actores no-estatales en la política internacional (Ardila, 2009). En este sentido, el Estado colombiano, así como los partidos políticos, hacen lo posible por llevar los incentivos al terreno transnacional (Anónimo, 2017).

Los comportamientos electorales de los migrantes colombianos en su país de origen, tienden a seguir el mismo patrón en cuanto a prácticas de votación en el exterior (Guarnizo, Portes, Haller, 2003). Existe una tendencia marcada de baja participación electoral en Colombia, caracterizada por la abstención (González, 2010). Consecuentemente, "es previsible que el comportamiento electoral de los emigrantes tenga una tendencia abstencionista" (ibidem, p. 69). Además, estudios previos sugieren que el voto de los expatriados tiene una connotación de tipo simbólica y no de efecto real sobre la política del país de origen (Boccagni, Ramírez, 2013); no fue hasta 1991, cuando se empezó a conceder derechos de participación a los connacionales tales como la doble ciudadanía, el voto para representantes del Senado y la dotación de circunscripciones especiales (Ardila, 2009; Palop-García, 2017).

Los procesos de globalización y de inclusión a connacionales en el exterior dieron paso a una "internacionalización posible de la paz" y de "diplomacias alternativas y paralelas" proponiendo la intervención de diversos actores en la realidad nacional (Ardila, Cardona, Ramírez, 2005). Así, desde la iniciativa emigrante, por medio de la participación electoral en el exterior, se han promovido derechos como la doble ciudadanía, el voto en elecciones legislativas y la elección de un representante en el exterior (Jones-Correa, 1998; Bermúdez, Lafleur, 2015). La representación se convirtió en una necesidad importante para los connacionales viviendo en el extranjero. Sin embargo, las motivaciones de patriotismo, civismo y sentimientos hacia el país de origen parecen ser más importantes que el contenido de los referéndums o la agenda política presidencial (Boccagni, 2011).

Es válido mencionar que la experiencia electoral previa ha contribuido con el aumento de oportunidades de participación desde el exterior para los propios emigrantes. Fue para el Plebiscito de Reforma Constitucional de 1990, donde los propios colombianos no-residentes se organizaron para pedirle al Estado colombiano, la doble ciudadanía (Bermúdez, Lafleur, 2015). Para los electores migrantes, las votaciones han tenido un impacto mayor que simplemente el proceso electoral, pues son oportunidades para encontrarse con otros connacionales y al mismo tiempo, buscar reconocimiento público (Boccagni, Ramírez, 2013). De ahí que se plantee la siguiente hipótesis:

H3: Los colombianos residentes en Guayaquil con (a) experiencia de votación previa en Colombia, (b) que indican la necesidad de obtener curules en la legislatura de origen, y (c) que han participado con mayor 
frecuencia en elecciones colombianas desde el exterior, tienen mayores probabilidades de votar en el Plebiscito de la Paz.

\section{Metodología}

La metodología utilizada en esta investigación se basó en el trabajo de campo de una muestra a conveniencia de colombianos residentes en Guayaquil (Ecuador). El objetivo fue conocer la opinión de colombianos residentes en Guayaquil en relación al Plebiscito de Paz del 2 de octubre, si (no) se acercaron a votar y cuáles fueron las razones. Se tomaron en cuenta entrevistas de diseño semi-estructurado a actores clave de las entidades gubernamentales relacionadas al voto extraterritorial, principalmente cónsules y funcionarios públicos colombianos; esto con la meta no solo de diseñar y refinar el instrumento de medición (encuesta), sino también de poder aproximarse a los encuestados siguiendo los protocolos establecidos por el gobierno colombiano para el voto extraterritorial de 2016.

Para el diseño de la encuesta, se trabajó con el marco teórico del transnacionalismo político, particularmente en relación a tres dimensiones: (1) perfil socio-demográfico, (2) compromiso migrante, y (3) comportamiento electoral. En este sentido, el cuestionario fue desarrollado para analizar las hipótesis arriba formuladas.

Días previos al trabajo de campo se realizó una prueba ex-ante con 25 cuestionarios, con el fin de detectar posibles desviaciones y errores. La unidad de análisis -colombianos residentes en Guayaquil- tenía que cumplir con una sola condición al momento de participar en la encuesta: tener un año o más residiendo en Ecuador.

Se utilizó una técnica de muestreo a conveniencia usualmente utilizada en este tipo de investigaciones, en donde la unidad de análisis es difícil de encontrar para ser encuestada, en un espacio y tiempo específicos. Se recurrió a los espacios abiertos, públicos y concurridos de la ciudad, tales como: parques, plazas, calles comerciales y centros de comercio.

Las encuestas fueron conducidas por un equipo de encuestadores calificados y entrenados para la ocasión, vinculados a la Universidad Casa Grande, coordinados y dirigidos por los autores de esta investigación. La tabulación de los datos fue realizada por el equipo colaborador utilizando el programa informático SPSS (versión 23). Un total de 590 respuestas fueron válidas en este proceso.

El trabajo de campo se realizó dentro de las áreas urbanas de Guayaquil. La primera pregunta que hicieron los encuestadores a las personas seleccionadas fue si su residencia habitual se encontraba en Ecuador, sin pasar la encuesta a aquellos ciudadanos que respondieron negativamente. La encuesta se 
distribuyó proporcionalmente durante las tres semanas post-electorales; por consiguiente, el número de cuestionarios fue similar en los diferentes días del trabajo de campo.

La encuesta utilizada se estructuró a partir de diferentes artículos previamente discutidos (Boccagni, 2011; Boccagni, Ramírez, 2013; Guarnizo, Portes, Haller, 2003; Escobar, Arana, Mccann, 2014; Portes, 2005; Umpierrez de Reguero, Dandoy, Palma, 2017). Las preguntas se refirieron a diferentes dimensiones del transnacionalismo político y comportamiento electoral. Se subdividió el cuestionario en relación a las tres secciones arriba indicadas. El primer bloque se usó para recopilar las características, actitudes y conocimiento acerca del compromiso migrante de los encuestados. La sección subsiguiente se centró en cuestiones institucionales, en específico del sistema electoral y propias del Plebiscito de la Paz. Finalmente, el tercer bloque incluyó diferentes características socio-demográficas de los encuestados.

En la encuesta, se usaron preguntas dicotómicas, con respuestas de tipo "sí" y "no". También se utilizaron preguntas con respuestas ordinales, por ejemplo de frecuencia o de escala de Likert, así como una pregunta abierta sobre las observaciones que los encuestados tenían sobre el Plebiscito de la Paz.

Dado que no se conocía el número exacto de colombianos en Guayaquil al momento de decidir la muestra, se trabajó con las cifras estimadas a nivel nacional, del censo internacional de Naciones Unidas. En respuesta, se realizó una estimación de este universo y un cálculo de error aproximado, que solo sirvió para una orientación metodológica del artículo. Así, según Naciones Unidas, el número estimado de colombianos en Ecuador para 2015 fue de 194.733 ciudadanos. Tomando esta cifra como referencia, el error de muestreo consistente con el número de respuestas válidas obtenidas, para un nivel de confianza del 95\%, fue de $\pm 4.04 \%$.

El análisis estadístico de los datos se realizó usando el programa informático SPSS. Se trabajó con análisis de regresión logística binaria para conocer los determinantes de la participación electoral, y análisis de componentes principales y diferencia de medias para entender en base a qué motivaciones se estructuraron las percepciones de (no) voto en relación al Plebiscito de Paz.

Para el análisis de regresión, la variable dependiente fue la participación de los encuestados en las elecciones del 2 de octubre de 2016 (Gráfico 1). El porcentaje de votación en la encuesta fue casi el doble que el total de votos válidos de la circunscripción internacional. De los 599.026 colombianos no-residentes registrados por las entidades electorales, solo el 13,6\% votó en el Plebiscito de la Paz del 2 de octubre de 2016 (El Tiempo, 03.10.2016). En consecuencia, realizar procesos de post-estratificación de muestra no fue productivo. 


\section{Gráfico 1. ¿Votó en el Plebiscito de la Paz?}

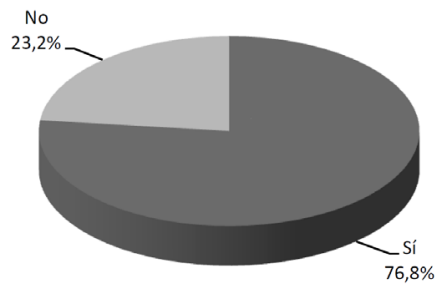

$N=590$.

Las variables independientes fueron nominales, al menos en su mayoría (Tabla 2). De hecho, un $90 \%$ de las variables se transformaron en dummy, codificando una respuesta de valor mínimo $<<0>>$ a máximo $<<1>>$. También, la edad y los años de residencia de los encuestados que originalmente eran variables en escala (razón), se las transformó en variables ordinales, agrupándolas en tramos para una mejor interpretación del ejercicio estadístico.

Tabla 2. Frecuencias y porcentajes de las variables independientes

\begin{tabular}{|c|c|c|c|c|}
\hline Dimensión & Variable & Categorías & $\mathbf{N}$ & $\%$ \\
\hline \multirow{14}{*}{$\begin{array}{l}\text { Perfil socio- } \\
\text { demográfico }\end{array}$} & \multirow{2}{*}{ Área de procedencia } & Rural (Colombia) & 83 & 14,1 \\
\hline & & Urbano (Colombia) & 507 & 85,9 \\
\hline & \multirow{2}{*}{ Género } & Masculino & 298 & 50,5 \\
\hline & & Femenino & 292 & 49,5 \\
\hline & \multirow{4}{*}{ Edad (tramos) } & 18-24 años & 206 & 34,9 \\
\hline & & 25-34 años & 140 & 23,7 \\
\hline & & 35-50 años & 148 & 25,1 \\
\hline & & $>50$ años & 96 & 16,3 \\
\hline & \multirow{3}{*}{ Años de residencia (tramos) } & 1-9 años de residencia & 209 & 35,4 \\
\hline & & $\begin{array}{l}10-19 \text { años de } \\
\text { residencia }\end{array}$ & 264 & 44,7 \\
\hline & & $\begin{array}{l}20 \text { o más años de } \\
\text { residencia }\end{array}$ & 117 & 19,8 \\
\hline & \multirow{3}{*}{ Estatus legal } & Refugiado & 53 & 9 \\
\hline & & $\begin{array}{l}\text { Visa temporal o } \\
\text { renovando permiso }\end{array}$ & 140 & 23,7 \\
\hline & & Residencia permanente & 397 & 67,3 \\
\hline
\end{tabular}




\begin{tabular}{|c|c|c|c|c|}
\hline \multirow{8}{*}{$\begin{array}{l}\text { Compromiso } \\
\text { migrante }\end{array}$} & \multirow{2}{*}{$\begin{array}{l}\text { Doble ciudadanía } \\
\text { (colombiano-ecuatoriano) }\end{array}$} & No & 408 & 69,2 \\
\hline & & Sí & 182 & 30,8 \\
\hline & \multirow{2}{*}{$\begin{array}{l}\text { Filiación política (en } \\
\text { Colombia) }\end{array}$} & No & 560 & 94,9 \\
\hline & & Sí & 30 & 5,1 \\
\hline & \multirow{2}{*}{$\begin{array}{l}\text { Asociación civil de } \\
\text { migrantes colombianos }\end{array}$} & No & 546 & 92,5 \\
\hline & & Sí & 44 & 7,5 \\
\hline & \multirow{2}{*}{ Retorno } & No & 446 & 75,6 \\
\hline & & Sí & 144 & 24,4 \\
\hline \multirow{8}{*}{$\begin{array}{l}\text { Comportami- } \\
\text { ento electoral }\end{array}$} & \multirow{2}{*}{$\begin{array}{l}\text { Experiencia electoral (en } \\
\text { Colombia) }\end{array}$} & No & 282 & 47,8 \\
\hline & & Sí & 308 & 52,2 \\
\hline & \multirow{2}{*}{$\begin{array}{l}\text { Representación especial de } \\
\text { emigrantes }\end{array}$} & No & 106 & 18 \\
\hline & & Sí & 484 & 82 \\
\hline & \multirow{2}{*}{$\begin{array}{l}\text { Seguimiento diálogos de } \\
\text { paz }\end{array}$} & No & 209 & 35,4 \\
\hline & & Sí & 381 & 64,6 \\
\hline & \multirow{2}{*}{$\begin{array}{l}\text { Preocupación Elecciones } \\
2018\end{array}$} & No & 482 & 81,7 \\
\hline & & Sí & 108 & 18,3 \\
\hline
\end{tabular}

$N=590$.

\section{Resultados}

Esta sección intenta responder a las siguientes dos preguntas: (1) ¿Por qué los encuestados (no) votaron en el Plebiscito de la Paz? y (2) iqué determinantes incidieron más en su respuesta? En otras palabras, se presenta evidencia, de los motivos asociados al uso del voto extraterritorial, pero directamente orientados a la discusión sobre la eficiencia, credibilidad y mensaje del Plebiscito de la Paz del 2 de octubre de 2016, y se caracteriza al potencial votante y analiza cuáles atributos, características, valores y actitudes incidieron en la decisión de participar en este proceso electoral.

\subsection{Motivos de participación}

En la encuesta se les dio a los encuestados enunciados con posibles motivos para (no) votar en el Plebiscito de la Paz. Estos siete enunciados (ver Tabla 3) fueron codificados ex ante como escala de Likert. Es decir, los colombianos residentes en Ecuador tenían que responder si estaban: totalmente en desacuerdo (1), en desacuerdo (2), indiferentes (3), de acuerdo (4) y totalmente de acuerdo (5), frente a cada enunciado.

Pese a que la media señala indiferencia por parte de los colombianos residentes en Ecuador con el proceso en análisis, por la desviación estándar se puede inferir que la mayoría de respuestas oscila entre en desacuerdo y de acuerdo. 


\section{Tabla 3. Estadísticos descriptivos de los posibles motivos de participación en el Plebiscito}

\begin{tabular}{|c|c|c|}
\hline & Media & Desv. Est. \\
\hline $\begin{array}{l}\mathrm{X}_{1} \text { : Otro intento fallido por parte del Gobierno de Colombia de acabar } \\
\text { con el conflicto armado }\end{array}$ & 2,87 & 1,01 \\
\hline $\mathrm{X}_{2}$ : Son mis derechos como colombiano/a a participar de esta decisión & 3,47 & 1,04 \\
\hline $\begin{array}{l}\mathrm{X}_{3} \text { : Es la mejor manera de acabar el conflicto armado entre Colombia } \\
\text { y la guerrilla }\end{array}$ & 3,16 & 1,09 \\
\hline $\mathrm{X}_{4}$ : Me devuelve la esperanza de retornar a Colombia a vivir & 3,01 & 0,99 \\
\hline $\mathrm{X}_{5}$ : Es una "pantomima" o "show" del Gobierno de Colombia & 3,02 & 1,01 \\
\hline $\mathrm{X}_{6}$ : Deber cívico con mi patria & 3,16 & 1,06 \\
\hline $\mathrm{X}_{7}$ : El acuerdo de paz no es beneficioso para el futuro de Colombia & 3,06 & 1,11 \\
\hline
\end{tabular}

El análisis de componentes principales se realizó examinando la bondad de ajuste suponiendo que la relación observada entre las variables puede atribuirse a factores comunes. En consecuencia, las correlaciones entre las variables independientes $\left(\mathrm{X}_{1}: \mathrm{X}_{7}\right)$ pueden deducirse a partir de las correlaciones estimadas entre las variables y los dos factores extraídos. El modelo factorial es adecuado debido que no se tienen residuos significativos. La prueba de Kaymer, Meyer y Olkin (KMO) ofreció un coeficiente razonable para la realización de este ejercicio estadístico $(\mathrm{KMO}=0,613)$. Todas las dimensiones utilizadas pueden ser consideradas como sólidos determinantes de las percepciones (positivas y negativas) de los encuestados sobre el Plebiscito de la Paz (Tabla 4). Los coeficientes Alfa de Cronbach de 0,720 para el componente 1 (percepciones positivas) y 0,641 para el componente 2 (percepciones negativas) señalan que las variables creadas tienen una alta/moderada fiabilidad para responder a la primera pregunta planteada al inicio de la sección de resultados. En efecto, este modelo de análisis de componentes principales cumple con las condiciones de parsimonia e interpretabilidad.

Tabla 4. Matriz de componentes rotados

\begin{tabular}{|c|c|c|}
\hline & \multicolumn{2}{|c|}{ Componentes } \\
\hline & 1 & 2 \\
\hline & $\begin{array}{l}\text { Percepciones } \\
\text { positivas }\end{array}$ & $\begin{array}{l}\text { Percepciones } \\
\text { negativas }\end{array}$ \\
\hline $\begin{array}{l}\mathrm{X}_{1} \text { : Es la mejor manera de acabar el conflicto armado entre } \\
\text { Colombia y la guerrilla }\end{array}$ & 0,814 & $-0,126$ \\
\hline $\mathrm{X}_{2}:$ Me devuelve la esperanza de retornar a Colombia a vivir & 0,788 & $-0,071$ \\
\hline $\begin{array}{l}\mathrm{X}_{3}: \text { Son mis derechos como colombiano/a a participar de esta } \\
\text { decisión }\end{array}$ & 0,716 & 0,393 \\
\hline $\mathrm{X}_{4}$ : Deber cívico con mi patria & 0,602 & 0,172 \\
\hline
\end{tabular}




\begin{tabular}{l|c|c}
\hline$X_{5}:$ Es una "pantomima" o "show" del Gobierno de Colombia & $-0,041$ & 0,850 \\
\hline$X_{6}:$ El acuerdo de paz no es beneficioso para el futuro de Colombia & 0,011 & 0,795 \\
\hline$X_{7}:$ Otro intento fallido por parte del Gobierno de Colombia de & 0,152 & 0,595 \\
\hline
\end{tabular}

Las pruebas de diferencia de medias muestran que la variable dependiente seleccionada en este artículo $\left(Y_{1}\right.$ : participación electoral en el Plebiscito de la Paz) está directamente correlacionada con el primer componente (percepción positiva). El segundo ejercicio de diferencia de medias no es estadísticamente significativo (ver Tabla 5). La prueba de Levene establece que las varianzas no son iguales en las dos pruebas.

\section{Tabla 5. Diferencias de medias (participación electoral en el Plebiscito de la Paz} y los componentes creados)

\begin{tabular}{l|c|c|c|c}
\hline & $\mathrm{t}$ & Sig. (bilateral) & $\begin{array}{c}\text { Diferencia de } \\
\text { medias }\end{array}$ & Error estándar \\
\hline Percepción positiva & $-3,347$ & 0,001 & $-0,282$ & 0,084 \\
\hline Percepción negativa & $-1,338$ & 0,183 & $-0,125$ & 0,093 \\
\hline
\end{tabular}

\subsection{Determinantes de la participación electoral transnacional}

Para explicar la participación electoral de colombianos residentes en Guayaquil (Ecuador) en el Plebiscito de la Paz de octubre de 2016, se corrieron dos modelos de regresión logística binaria (ver Tabla 6). En el primero, se incluyó todas las variables independientes indicadas en la metodología de este trabajo. En el segundo modelo, se incorporaron las mismas variables más las dos variables creadas con el análisis de componentes principales: percepciones positivas y negativas.

La edad es estadísticamente significativa en ambos modelos. La participación electoral en el Plebiscito de la Paz está inversamente correlacionada a los encuestados de 25 a 50 años, en relación a los jóvenes (de 18 a 24 años) que fue utilizada como categoría de referencia. Aunque el planteamiento de algunos autores al respecto (e.g. Ayla-Lastra, 2006) puede ser opuesto a estos resultados, se detecta que los jóvenes pueden ser más sensibles a procesos electorales específicos como el Plebiscito de la Paz. Después de todo son las nuevas generaciones quienes impulsan el post-materialismo (Inglehart, Norris, 2017). El resto de variables de la dimensión perfil socio-demográfico, al igual que las cuatro variables independientes asociadas al compromiso migrante, no inciden sobre la variable de respuesta en ninguno de los dos modelos.

En relación a la tercera dimensión -comportamiento electoral-, los modelos demuestran que a mayor votación previa en Colombia por parte del 
migrante, son mayores las probabilidades de participación en elecciones futuras desde el exterior. Este resultado no es sorprendente. De hecho, la relación entre experiencia en elecciones anteriores (en Colombia) y la participación electoral en el exterior, coincide con la literatura previa. Se puede estimar que el comportamiento de los migrantes colombianos con respecto a su participación política, suele replicarse -quienes sufragaron de manera regular en su país de origen, a menudo repiten este patrón en el país de residencia (Guarnizo, Portes, Haller, 2003)-. De hecho, la experiencia electoral previa ha contribuido con el incremento de oportunidades de participación desde el exterior para los propios emigrantes (Bermúdez, Lafleur, 2015).

Ahora bien, las variables contextuales asociadas a la dimensión de comportamiento electoral -seguimiento al Acuerdo de Paz y la preocupación por elecciones de 2018- son evidencias para reformular el argumento sobre a mayor integración del migrante en el país de residencia, menor interés por informarse sobre la situación de su país de origen (Bauböck, 2003). Con el avance exponencial de la tecnología en la comunicación, los migrantes no solo pueden informarse mejor de lo que ocurre en su país, como lo demuestra el $64,6 \%$ de la muestra que declaró haber seguido los diálogos del Acuerdo de Paz entre el Gobierno y las guerrillas (ver Tabla 2), sino también hay un porcentaje considerable que declaró estar preocupado por las elecciones futuras en Colombia, al momento de llenar la encuesta. Para octubre y noviembre de 2016, las elecciones más cercanas eran las presidenciales y las legislativas de 2018. Al estar directamente correlacionada la participación electoral en el exterior con las variables seguimiento al Acuerdo de Paz y preocupación por las elecciones 2018, se puede inferir que al menos el colombiano que reside en Ecuador percibe a la realidad política y social de Colombia muy de cerca. En promedio, este perfil es el de un migrante que puede clasificarse como preocupado o interesado por lo que ocurre en su país de origen, lo que tiene bastante sentido, particularmente si esta discusión de resultados se enmarca en los debates abiertos del mundo académico sobre transnacionalismo político e integración.

En el Modelo 2, el coeficiente $b$ de las percepciones positivas hace que nuevamente se rechace la hipótesis nula. En respuesta, mientras más positivas son las percepciones de los colombianos residentes en Ecuador, mayores sus probabilidades de participar electoralmente desde el exterior. Este resultado, posiblemente, se lo pueda asociar a la ideología del encuestado, a la aprobación de los colombianos residentes en Ecuador del gobierno de Juan Manuel Santos, pero también a la constitución misma del componente. Dentro de los enunciados que formaron el primer componente, se puede encontrar una asociación directa con el sentimiento de patriotismo, cívica, inclusive la obligación de ejercer derechos político-electorales por la propia 
configuración del sistema electoral (relación previamente ya explorada por Boccagni, Ramírez, 2013). Bajo este contexto, es habitual pensar que quienes tengan el derecho al voto como primer motivo para ir a sufragar sean quienes tengan más probabilidades de participar en elecciones de su país de origen en el exterior.

Tabla 6. Regresión logística binaria

\begin{tabular}{|c|c|c|}
\hline & Modelo 1 & Modelo 2 \\
\hline & b (Error Estándar) & b (Error Estándar) \\
\hline Área de procedencia & $0,091(0,314)$ & $0,043(0,319)$ \\
\hline Género & $-0,359(0,213)$ & $-0,323(0,216)$ \\
\hline 18-24 años & . & . . \\
\hline 25-34 años & $-0,722 *(0,322)$ & $-0,717 *(0,324)$ \\
\hline $35-50$ años & $-0,773 *(0,328)$ & $-0,769 * *(0,332)$ \\
\hline$>50$ años & $-0,465(0,375)$ & $-0,451(0,379)$ \\
\hline 1-9 años de residencia & . & . . \\
\hline 10-19 años de residencia & $0,244(0,257)$ & $0,292(0,262)$ \\
\hline 20 o más años de residencia & $0,043(0,347)$ & $0,107(0,354)$ \\
\hline Refugiado & $-0,171(0,421)$ & $-0,164(0,424)$ \\
\hline Visa temporal o renovando permiso & $-0,069(0,266)$ & $-0,071(0,269)$ \\
\hline Residencia permanente & . . & . . \\
\hline Doble ciudadanía (colombo-ecuatoriano) & $-0,143(0,255)$ & $-0,235(0,260)$ \\
\hline Filiación política de Colombia & $0,51(0,446)$ & $0,466(0,454)$ \\
\hline Asociación civil de migrantes colombianos & $0,333(0,398)$ & $0,347(0,404)$ \\
\hline Esperanza de retorno & $-0,141(0,262)$ & $-0,080(0,264)$ \\
\hline Experiencia en elecciones anteriores (en Colombia) & $1,684 * * *(0,288)$ & $1,626 * * *(0,292)$ \\
\hline Representación especial de emigrantes & $0,408(0,293)$ & $0,302(0,297)$ \\
\hline Seguimiento al Acuerdo de Paz & $0,889 * * *(0,246)$ & $0,829 * * *(0,248)$ \\
\hline Preocupación elecciones 2018 & $0,604 *(0,252)$ & $0,572 *(0,254)$ \\
\hline Componente 1 (Percepciones positivas) & & $0,376 *(0,149)$ \\
\hline Componente 2 (Percepciones negativas) & & $0,112(0,135)$ \\
\hline Constante & $-2,867 * * *(0,537)$ & $-4,267 * * *(0,806)$ \\
\hline Prueba Ji-cuadrado & $<0,001$ & $<0,001$ \\
\hline Prueba Cox-Schnel & 0,130 & 0,141 \\
\hline Prueba Nagelkerke & 0,196 & 0,212 \\
\hline Clasificación & $67,10 \%$ & $67,80 \%$ \\
\hline $\mathrm{N}$ & 590 & 590 \\
\hline Curva ROC (Coeficiente) & 0,743 & 0,757 \\
\hline
\end{tabular}

$* p<0,05, * * p<0,01 ; * * *<<0,001$ 
La bondad de ajuste de los dos modelos presentados es aceptable. Si bien no es una regresión de mínimos cuadrados donde el $\mathrm{R}^{2}$ es fundamental para identificar cuánto explica el modelo, y por tanto, resultados como los que se pueden observar en la Tabla 6 pueden resultar insuficientes; para una regresión logística binaria es usual y admisible reportar (pseudo) $\mathrm{R}^{2}$ bajos. Las pruebas de especificidad y sensibilidad del modelo confirman que efectivamente el modelo presentado es viable. Los porcentajes globales de clasificación son de $67,1 \%$ y $67,8 \%$, respectivamente; las pruebas de ji-cuadrado en ambos modelos son significativas a un nivel menor o igual a 0,001. Adicionalmente, se corrió la curva de ROC por cada modelo, entre los valores predichos y la variable de respuesta, dando como resultado coeficientes de 0,743 y 0,757 ; lo que confirma una vez más la precisión de los ejercicios estadísticos.

\section{Conclusiones}

En este artículo se examinó la relación entre el voto extraterritorial y el referéndum como mecanismo de democracia directa. En particular, se halló los determinantes de votación en el Plebiscito de la Paz del 2 de octubre de 2016 e identificó los motivos más frecuentes para (no) votar en este proceso electoral desde el exterior, agrupados en dos componentes -(1) percepciones positivas y (2) percepciones negativas-.

El diseño de investigación empleado fue observacional, de tipo descriptivo y explicativo. Los datos provinieron de 590 respuestas de emigrantes y refugiados colombianos en Ecuador, y se estructuraron a partir de sus percepciones sobre el Plebiscito de la Paz del 2 de octubre de 2016. Las variables con las que se construyó la evidencia empírica se articularon a partir de las siguientes tres dimensiones: (1) perfil socio-demográfico, (2) compromiso migrante y (3) comportamiento electoral.

La prueba de diferencias de medias que se realizó con los dos componentes y la variable dependiente $\left(\mathrm{Y}_{1}\right)$ participación electoral en el exterior, reportó únicamente una relación estadísticamente significativa cuando las percepciones son positivas. En otras palabras, cuando los colombianos residentes en Ecuador están de acuerdo y totalmente de acuerdo con los enunciados vinculados al primer componente (e.g. "me devuelve la esperanza de retornar a Colombia a vivir" o "son mis derechos como colombiano/a participar de esta decisión"), existen mayores probabilidades de participación electoral en el exterior. Ahora bien, no se rechazó la hipótesis nula entre el segundo componente asociado a percepciones negativas y la participación electoral en el exterior.

Por su parte, la dimensión de comportamiento electoral es la que más explica y pesa en los modelos logísticos binarios de participación electoral en el exterior (ver Tabla 6). De las cuatro variables relacionadas con esta dimensión, 
en tres ocasiones la hipótesis nula se rechaza en aceptación de la hipótesis alternativa que establece una relación positiva con la variable dependiente. En otras palabras, tanto la experiencia en elecciones anteriores (en Colombia), el seguimiento a los Acuerdos de Paz como la preocupación por las elecciones de 2018 son estadísticamente significativas. Solo la representación especial de emigrantes o también entendida como la necesidad de reservar curules para la representación política en la legislatura (Umpierrez de Reguero, Dandoy, Palma, 2017), no incide en la participación electoral en el exterior.

Las dimensiones del perfil socio-demográfico y compromiso migrante no inciden en la participación electoral en el exterior, salvo los tramos de edad, particularmente los encuestados de 25 a 50 años que tienen una relación inversa con la variable dependiente, teniendo como categoría de referencia a los jóvenes (de 18 a 24 años). En este sentido, se puede estimar que los jóvenes son quienes tienen mayor propensión a sufragar en elecciones como la del Plebiscito de Paz de 2016.

\section{Referencias}

ACNUR. Tendencias Globales. Desplazamiento forzado en 2016. Geneva: ACNUR, 2017.

Alto Comisionado Para La Paz. Acuerdo final para la terminación del conflicto y la construcción de una paz estable y duradera. 24.11.2016. Disponible en: < http:// www.altocomisionadoparalapaz.gov.co/procesos-y-conversa ciones/Documentos\%20 compartidos/24-11-2016NuevoAcuerdoFinal.pdf>.

ANÓNIMO. Entrevista Oficial Diplomático, comunicación personal. Octubre 2017. ARDILA, Martha. Actores No Gubernamentales Y Política Exterior. A propósito del sector académico y el diseño de la política exterior migratoria colombiana. Colombia Internacional, n. 69, p. 108-123, 2009.

ARDILA, Martha; CARDONA, Diego; RAMÍREZ, Socorro. Colombia y su política exterior en el siglo XXI. Bogotá: Friedrich-Ebert-Stiftung, 2005.

AYSA-LASTRA, Martha. Perfil Sociodemográfico de los Emigrantes Colombianos a Estados Unidos. Presentado en Cátedra de las Américas, Barranquilla, Colombia, 2006.

BARRY, Kim. Home and away: the construction of citizenship in an emigration context. New York University Law Review, n. 1, p. 11-59, 2006.

BAUBÖCK, Rainer. Towards a Political Theory of Migrant Transnationalism. The International Migration Review, n. 3, p. 700-723, 2003.

BAUBÖCK, Rainer. Stakeholder citizenship and transnational political participation: a normative evaluation of external voting. Fordham Law Review, v. 75, p. 23932447, 2007.

BELCHIOR, Ana Maria; AZEVEDO, Joana; LISI, Marco; ABRANTES, Manuel. Contextual reasons for emigrants' electoral participation in home country elections: the Portuguese case. Journal of Contemporary European Studies, p. 1-18, 2017. 
BERG, Ulla Dalum; TAMAGNO, Carla. El Quinto Suyo from above and from below: state agency and transnational political practices among Peruvian migrants in the US and Europe. Latino Studies, v. 4, n. 3, p. 258-281, 2006.

BERMÚDEZ, Anastasia. El vínculo de los estados con sus ciudadanos en el exterior: el Caso de los migrantes colombianos en Europa. Revista de la Asociación Española de Americanistas, n. 13, p. 1-20, 2014.

BERMÚDEZ, Anastasia; LAFLEUR, Jean-Michel. Diaspora Contributions to Democratic Processes at Home: The External Vote of Andean Migrants. Sociologie du vote à distance. Provenza: Congrès AFSP Aix, 2015.

BOCCAGNI, Paolo. Reminiscences, Patriotism, Participation: Approaching External Voting in Ecuadorian Immigration to Italy. International Migration, v. 49, n. 3, p. 76-98, 2011.

BOCCAGNI, Paolo; RAMÍREZ, Jacques. Building Democracy or Reproducing 'Ecuadoreanness'? A Transnational Exploration of Ecuadorean Migrants' External Voting. Journal of Latin American Studies, v. 45, p. 721-750, 2013.

BRAND, Laurie A. Authoritarian states and voting from abroad: North African experiences. Comparative Politics, 2010, v. 43, n. 1, p. 81-99.

CALDERÓN-CHELIUS, Leticia. El estudio de la dimensión política dentro del proceso migratorio. Sociológica, v. 21, n. 60, p. 43-73, 2006.

CARREÑO-MALAVER, Ángela. Refugiados colombianos en Venezuela: Quince años en búsqueda de protección. Memorias, n. 24, p 98-124, 2014.

CLAVIJO-PADILLA, Janneth. Los colombianos en el exterior en la política migratoria reciente: análisis del programa Colombia Nos Une. Revista Chilena de Derecho y Ciencia Política, v. 4, n. 3, p. 85-121, 2013.

COLLYER, Michael. A geography of extra-territorial citizenship: Explanations of external voting. Migration Studies, v. 2, n. 1, p. 55-72, 2014 a.

COLLYER, Michael. Inside out? Directly elected 'special representation' of emigrants in national legislatures and the role of popular sovereignty. Political Geography, v. 41, p. 64-73, 2014b.

COLLYER, Michael; VATHI, Zana. Patterns of Extra-territorial Voting. Working Paper T 22, Development Research Centre on Migration, Globalisation and Poverty, 2007.

EL TIEMPO. En el exterior, mayoría de colombianos apoyaron el 'Sí' en plebiscito. 03.10.2016. Disponible en: <http://www.eltiempo.com/politica/proceso-depaz/resultados-plebiscito-en-el-exterior-31845>.

ERLINGSSON, Hafthor; TUMAN, John P. External Voting Rights in Latin America and the Caribbean: The Influence of Remittances, Globalization, and Partisan Control. Latin American Policy, v. 8, n. 2, p. 295-312, 2017.

ESCOBAR, Cristina. Dual citizenship and political participation: migrants in the interplay of United States and Colombian politics. In: OBOLER, Suzanne (ed.). Latinos and Citizenship: The Dilemma of Belonging. New York: Palgrave, 2006, p. 113-141. 
ESCOBAR, Cristina. Extraterritorial Political Rights and Dual Citizenship in Latin America. Latin American Research Review, v. 42, n. 3, p. 43-75, 2007.

ESCOBAR, Cristina; ARANA, Renelinda; MCCANN, James. Assessing Candidates at Home and Abroad: A Comparative Analysis of Colombian Expatriates in the 2010 Presidential Elections. Latin American Politics and Society, v. 56, n. 2, p. 115-140, 2014.

GAMLEN, Alan. The impacts of extra-territorial voting: Swings, interregnums and feedback effects in New Zealand elections from 1914 to 2011. Political Geography, v. 44, p. 1-8, 2015.

GARAY-SALAMANCA, Luis Jorge; MEDINA, María. La migración colombiana a España. El capítulo más reciente de una historia compartida. Ministerio de Trabajo e Inmigración, 2007.

GIORGULI-SAUCEDO, Silvia; ITZIGSOHN, José. Diferencias de género en la experiencia migratoria. Trasnacionalismo e incorporación de los migrantes latinos en Estados Unidos. Papeles de población, v. 12, n. 47, p. 9-37, 2006.

GONZÁLEZ, Olga. El voto de los colombianos en el exterior: elecciones entre disfuncionamientos y rebusque. Ciencia Política, n. 9, p. 62-77, 2010.

GUARNIZO, Luis Eduardo; SÁNCHEZ, Arturo; ROACH, Elizabeth. Mistrust, fragmented solidarity, and transnational migration: Colombians in New York City and Los Angeles. Ethnic and Racial Studies, v. 22, n. 2, p. 367-396, 1999.

GUARNIZO, Luis Eduardo; PORTES, Alejandro; HALLER, William. Assimilation and Transnationalism: Determinants of Transnational Political Action among Contemporary Migrants. American Journal of Sociology, v. 108, n. 6, p. 12111248, 2003.

INGLEHART, Ronald; NORRIS, Pippa. Trump and the populist authoritarian parties: the silent revolution in reverse. Perspectives on Politics, v. 15, n. 2, p. 443-454, 2017.

IDEA-IFE. El voto en el extranjero. El manual de IDEA Internacional. Stockholm: International IDEA, 2007.

JONES-CORREA, Michael. Between Two Nations: The Political Predicament of Latinos in New York City. Ithaca, NY: Cornell University Press, 1998.

LAFLEUR, Jean-Michel. Why do states enfranchise citizens abroad? Comparative insights from Mexico, Italy and Belgium. Global Networks, v. 11, p. 481-501, 2011.

LAFLEUR, Jean-Michel. Transnational politics and the state: The external voting rights of Diasporas. London: Routledge, 2013.

LAFLEUR, Jean-Michel. The enfranchisement of citizens abroad: variations and explanations. Democratization, v. 22, p. 840-860, 2015.

LEVITT, Peggy; DE LA DEHESA, Rafael. Transnational migration and the redefinition of the state: Variations and explanations. Ethnic and Racial Studies, v. 26, n. 4, p. 587-611, 2003.

LEVITT, Peggy; SCHILLER, Nina Glick. Conceptualizing simultaneity: A transnational social field perspective on society. International Migration Review, v. 38, n. 3, p. 1002-1039, 2004. 
LEVITT, Peggy; WATERS, Mary C. The Changing Face of Home: The Transnational Lives of the Second Generation. New York: Russell Sage Foundation, 2002.

LISI, Marco; BELCHIOR, Ana Maria; ABRANTES, Manuel; AZEVEDO, Joana. Out of Sight, Out of Mind? External Voting and the Political Representation of Portuguese Emigrants. Journal of South European Society and Politics, v. 20, p. 265-285, 2014.

LÓPEZ-GUERRA, Claudio. Should Expatriates Vote? Political Philosophy, v. 13, p. 216-234, 2005.

MARTINIELLO, Marco; LAFLEUR, Jean-Michel. Towards a Transatlantic Dialogue in the Study of Immigrant Political Transnationalism. Journal of Ethnic and Racial Studies, v. 31, n. 4, p.645-663, 2008.

ØSTERGAARD-NIELSEN, Eva. The politics of migrants' transnational political practices. International Migration Review, v. 37, p. 760-786, 2003.

ØSTERGAARD-NIELSEN, Eva. Sending Country Policies. In: GARCÉSMASCAREÑAS, Blanca; PENNINX, Rinus (eds.). Integration Processes and Policies in Europe. Springer International Publishing, p. 147-165, 2016.

ØSTERGAARD-NIELSEN, Eva; CIORNEI, Irina. Making the absent present: political parties and emigration issues in country of origin parliaments. Party Politics, p. 1-14, 2017.

PALOP-GARCÍA, Pau. Ausentes, pero representados: mecanismos institucionales de representación de emigrantes en América Latina y el Caribe. America Latina Hoy: Revista de Ciencias Sociales, n. 76, p. 15-34, 2017.

PALOP-GARCÍA, Pau; PEDROZA, Luicy. Beyond convergence: unveiling variations of external franchise in Latin America and the Caribbean from 1950 to 2015. Journal of Ethnic and Migration Studies, v. 43, n. 9, p. 1597-1616, 2017.

PORTES, Alejandro. Convergencias teóricas y evidencias empíricas en el estudio del transnacionalismo de los inmigrantes. Migración y Desarrollo, n. 4, p. 2-19, 2005.

PORTES, Alejandro; ESCOBAR, Cristina; WALTON, Alexandria. Organizaciones transnacionales de inmigrantes y desarrollo: un estudio comparativo. Migración y Desarrollo, n. 6, p. 3-44, 2006.

RAGAZZI, Francesco. A comparative analysis of diaspora politics. Political Geography, v. 41, p. 74-89, 2014.

RENSHON, Stanley. Dual Citizenship and American National Identity. Washington D.C.: Center for Immigration Studies, 2001.

RHODES, Sybil; HARUTYUNYAN, Arus. Extending Citizenship to Emigrants: Democratic Contestation and a New Global Norm. International Political Science Review, v. 31, p. 470-493, 2010.

ROBERTS, Bryan; FRANK, Reanne; LOZANO, Fernando-Asensio. Transnational migrant communities and Mexican migration to the US. Journal of Ethnic and Racial Studies, v. 22, n. 2, p. 238-266, 1999. 
RUBIO-MARIN, Ruth. Transnational politics and the democratic nation-state: normative challenges of expatriate voting and nationality retention of emigrants. New York University Law Review, v. 81, n. 1, p. 117-147, 2006.

TURCU, Anca; URBATSCH, Robert. Diffusion of Diaspora Enfranchisement Norms A Multinational Study. Comparative Political Studies, v. 48, n. 4, p. 407-437, 2014.

UMPIERREZ DE REGUERO, Sebastián; DANDOY, Régis; PALMA, Tatiana. Emigración y representación especial: Evidencia de los Ecuatorianos Residentes en el exterior. REMHU, Revista Interdisciplinar da Mobilidade Humana, v. 25, n. 50, p. 177-201, 2017.

UMPIERREZ DE REGUERO, Sebastián; GONZÁLEZ-PAREDES, Santiago; JARAALBA, Carol. Terremoto 16/A: Determinantes de la movilización de ayuda de los ciudadanos no-residentes hacia Ecuador. In: FRUGONE, Marcela; UMPIERREZ DE REGUERO, Sebastián; GARAY, Ignacio (eds.). Desastres y gestión de riesgos: desde un abordaje interdisciplinario. Guayaquil: Universidad Casa Grande, 2018.

United Nations Statistics Division. 2015 [consulta: 2017]. Disponible en: < https:// unstats.un.org/unsd/demographic/sources/census/censusdates.htm >.

Recibido para publicación en 16.02.2018

Aceptado para publicación en 29.05.2018

Received for publication in February $16^{\text {th }}, 2018$

Accepted for publication in May 29th, 2018

ISSN impresso 1980-8585

ISSN eletrônico 2237-9843

http://dx.doi.org/10.1590/1980-85852503880005410 